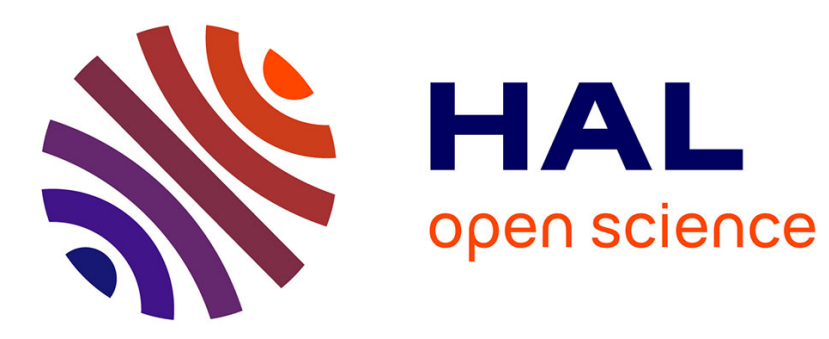

\title{
A quasi-optimal convergence result for fracture mechanics with XFEM
}

Elie Chahine, Patrick Laborde, Yves Renard

\section{To cite this version:}

Elie Chahine, Patrick Laborde, Yves Renard. A quasi-optimal convergence result for fracture mechanics with XFEM. Comptes rendus de l'Académie des sciences. Série I, Mathématique, 2006, 342, pp.527-532. 10.1016/j.crma.2006.02.002 . hal-00690581

\section{HAL Id: hal-00690581 \\ https://hal.science/hal-00690581}

Submitted on 7 Feb 2018

HAL is a multi-disciplinary open access archive for the deposit and dissemination of scientific research documents, whether they are published or not. The documents may come from teaching and research institutions in France or abroad, or from public or private research centers.
L'archive ouverte pluridisciplinaire HAL, est destinée au dépôt et à la diffusion de documents scientifiques de niveau recherche, publiés ou non, émanant des établissements d'enseignement et de recherche français ou étrangers, des laboratoires publics ou privés. 


\title{
A quasi-optimal convergence result for fracture mechanics with XFEM
}

\author{
Elie Chahine ${ }^{\mathrm{a}}$, Patrick Laborde ${ }^{\mathrm{b}}$, Yves Renard ${ }^{\mathrm{a}}$ \\ a MIP, CNRS UMR 5640, INSAT, 135, avenue de Rangueil, 31077 Toulouse cedex 4, France \\ ${ }^{\mathrm{b}}$ MIP, CNRS UMR 5640, UPS Toulouse 3, 118 route de Narbonne, 31062 Toulouse cedex 4, France
}

The aim of this Note is to give a convergence result for a variant of the eXtended Finite Element Method (XFEM) on cracked domains using a cut-off function to localize the singular enrichment area. The difficulty is caused by the discontinuity of the displacement field across the crack, but we prove that a quasi-optimal convergence rate holds in spite of the presence of elements cut by the crack. The global linear convergence rate is obtained by using an enriched linear finite element method.

Résultat de convergence quasi-optimal en mécanique de la rupture avec XFEM. Le but de cette Note est de donner un résultat de convergence pour une variante de la méthode XFEM (eXtended Finite Element Method) sur un domaine fissuré en utilisant une fonction cut-off pour localiser l'enrichissement par les fonctions singulières. La difficulté est causée par la discontinuité du champ de déplacement à travers la fissure, mais on montre une convergence quasi-optimale malgré la présence d'éléments coupés par la fissure. Le résultat de convergence globale linéaire est obtenu en utilisant une méthode d'éléments finis affines enrichis.

\section{Version française abrégée}

Les méthodes d'éléments finis classiques utilisées pour la modélisation des fissures, pour être efficaces, doivent respecter la géométrie de la fissure étudiée. Cette approche n'est pas très avantageuse dans la mesure où elle nécessite un grand nombre de degrés de liberté pour le raffinement en fond de fissure, ainsi qu'un remaillage lors de la propagation de la fissure. C'est la raison qui a motivé l'introduction de la méthode des éléments finis enrichis (XFEM : eXtended Finite Element Method) par Moës, Dolbow et Belytschko en 1999 (voir [11]). Celle-ci consiste à ajouter

E-mail addresses: elie.chahine@insa-toulouse.fr (E. Chahine), laborde@mip.ups-tlse.fr (P. Laborde), yves.renard@insa-toulouse.fr (Y. Renard). 
à la base éléments finis classique des fonctions singulières au voisinage de la pointe de la fissure, et une fonction discontinue tout au long de la fissure.

Le but de cette Note est de donner un résultat de convergence pour une variante de la méthode XFEM. Des résultats numériques ont montré que la méthode XFEM classique converge en $\sqrt{h}$, où $h$ est le pas du maillage. Ceci ne présente pas une amélioration de l'ordre de convergence de la solution de la méthode des éléments finis classique (voir $[8,13])$. Cette dernière permet même de réaliser une meilleure convergence pour le calcul des facteurs d'intensité de contraintes (voir [3]). Nous établissons, dans ce qui suit, que la variante proposée converge en $h$ pour des éléments finis affines. On considère, dans la Section 2, le problème d'élasticité linéaire (1) sur un domaine bidimensionnel borné et fissuré $\Omega$, et on suppose que le déplacement $u$ solution est décomposé comme en (2). Dans la section suivante, on définit un espace d'éléments finis affines enrichi par des fonctions singulières et une fonction discontinue sur une triangulation de $\bar{\Omega}$ (voir (4) et Fig. 1(b)). Dans la Section 4, le résultat de convergence est donné par le Théorème 4.1, suivi des étapes principales de la preuve. Le domaine $\Omega$ est divisé en deux suivant la fissure et son prolongement (Fig. 1(a)), ce qui permet de définir un opérateur d'interpolation (voir (11) et (12)) en utilisant un opérateur d'extension (10). Puis, on calcule les erreurs d'interpolation locales sur les élements totalement enrichis par la fonction discontinue $H$ (Lemme 4.2), sur l'élément contenant le fond de la fissure (Lemme 4.3), et sur les éléments partiellement enrichis par $H$ (Lemme 4.4).

\section{Introduction}

Classical finite element methods used for modeling crack propagation are subjected to several constraints: the mesh should match the crack geometry, should always evolve with the crack growth and should be refined near the crack tip. This motivated Moës, Dolbow and Belytschko to introduce an approach called XFEM (eXtended Finite Element Method) in 1999 (see [11]). The idea is to add singular functions to the finite element basis taking into account the singular behavior around the crack tip, and a step function modeling the discontinuity of the displacement field across the crack. This allows us to use a fixed mesh independent of the crack geometry. A better approximated solution, than the classical finite element one, can be obtained by choosing the appropriate singular enrichment (see [5]). For a two-dimensional crack, the singular enrichment functions are based on the exact solution given in $[9,10]$.

The aim of this Note is to give a convergence result for a variant of the XFEM. Numerical results showed that the convergence error of the XFEM is of order $\sqrt{h}$, where $h$ is the mesh parameter. This is not an improvement of the convergence order of the classical finite element method solution (see $[8,13]$ ). This later method can even realize better convergence results for the computation of the stress intensity factors (see [3]). We will prove, in what follows, that the convergence error of the proposed variant is of order $h$ for a linear finite element method. We consider in Section 2, the elasticity problem over a cracked bi-dimensional domain $\Omega$ and we suppose that the displacement field $u$ can be written as a sum of a singular part belonging to $H^{3 / 2-\epsilon}$ and a 'regular discontinuous' part (see [6]). In Section 3, we define an affine finite element space enriched by singular functions and by a discontinuous function over a triangulation of $\bar{\Omega}$. In Section 4, the main convergence result is given in Theorem 4.1 followed by the essential steps of the proof. Indeed, the domain $\Omega$ is divided into two subdomains which allow us to define an interpolation operator based on an extension operator. Then we compute, in separate lemmas, the local interpolation errors over the elements totally enriched by the discontinuous function $H$, over the element containing the crack tip and over the elements partially enriched by $H$.

\section{Model problem}

Let $\Omega$ be a bounded cracked domain in $\mathbb{R}^{2}$ and let $\Gamma_{C}$ be the crack which is assumed to be straight. We consider the linear elasticity problem on this domain for an isotropic material. The boundary of $\Omega$, denoted $\partial \Omega$, is partitioned into $\Gamma_{D}$ where a Dirichlet condition is applied, $\Gamma_{N}$ where a Neumann condition is applied, and $\Gamma_{C}$ (the crack) where we assume having a traction free condition.

Let $\vartheta=\left\{v \in H^{1}(\Omega) ; v=0\right.$ on $\left.\Gamma_{D}\right\}$ be the space of admissible displacements and

$$
a(u, v)=\int_{\Omega} \sigma(u): \varepsilon(u) \mathrm{d} x, \quad l(v)=\int_{\Omega} f \cdot v \mathrm{~d} x+\int_{\Gamma_{N}} g \cdot v \mathrm{~d} \Gamma, \quad \sigma(u)=\lambda \operatorname{tr} \varepsilon(u) I+2 \mu \varepsilon(u),
$$


with $\sigma(u)$ denoting the stress tensor, $\varepsilon(u)$ the linearized strain tensor, $g$ and $f$ some given densities on $\Gamma_{N}$ and $\Omega$ respectively, $\lambda>0$ and $\mu>0$ the Lamé coefficients. The problem can be written

$$
\text { find } u \in \vartheta \text { such that } a(u, v)=l(v) \quad \forall v \in \vartheta .
$$

We suppose that $f$ and $g$ are sufficiently smooth $\left(f \in H^{\epsilon}(\Omega)\right.$, and $g \in H^{1 / 2+\epsilon}\left(\Gamma_{N}\right)$ for some $\left.\epsilon \in\right] 0 ; 1 / 2[)$ such that the solution $u$ to the elasticity problem can be written as a sum of a singular part $u_{s}$ and a 'regular discontinuous' part satisfying (see [6]):

$$
u-r^{1 / 2}\left(c_{1} u_{\mathrm{I}}+c_{2} u_{\mathrm{II}}\right)=u-u_{s} \in H^{2+\epsilon}(\Omega),
$$

where $r$ denotes the distance to the crack tip. The normal (respectively tangential) component of the function $u_{\mathrm{I}}$ (respectively $u_{\mathrm{II}}$ ) is discontinuous along the crack. They both correspond to the well known I and II opening modes for a bi-dimensional crack (see $[9,10]$ ). The asymptotic displacement at the crack tip for these two modes is given in polar coordinates by

$$
u_{\mathrm{I}}=\frac{K_{\mathrm{I}}}{E} \sqrt{\frac{r}{2 \pi}}(1+v)\left(\begin{array}{c}
\cos \frac{\theta}{2}(3-4 v-\cos \theta) \\
\sin \frac{\theta}{2}(3-4 v-\cos \theta)
\end{array}\right), \quad u_{\mathrm{II}}=\frac{K_{\mathrm{II}}}{E} \sqrt{\frac{r}{2 \pi}}(1+v)\left(\begin{array}{c}
\sin \frac{\theta}{2}\left(C_{1}+2+\cos \theta\right) \\
\sin \frac{\theta}{2}\left(C_{1}-2+\cos \theta\right)
\end{array}\right),
$$

where $K_{\mathrm{I}}, K_{\mathrm{II}}$ denote the stress intensity factors, $v$ the Poisson ratio and $C_{1}=3-4 v$ in the plane stress problem. Note that $u_{\mathrm{I}}$ and $u_{\mathrm{II}}$ belong to $H^{3 / 2-\epsilon}(\Omega)$ for any $\epsilon>0$ (see [6]).

\section{XFEM: description and discretized problem}

The idea of XFEM is to use a classical finite element space enriched by some additional functions. These functions result from the product of global enrichment functions and some classical finite element functions (see [11]). We consider a linear Lagrange finite element method defined on a regular triangulation of the non-cracked domain $\bar{\Omega}$. The piecewise $P_{1}$ basis functions are denoted $\varphi_{1}, \ldots, \varphi_{N}$ ( $P_{1}$ is the set of degree one polynomials). The enriched space of the XFEM variant we propose is then

$$
\vartheta^{h}=\left\{v^{h}=\sum_{i \in I} a_{i} \varphi_{i}+\sum_{i \in I_{H}} b_{i} H \varphi_{i}+\sum_{j=1}^{4} c_{j} F_{j} \gamma ; a_{i} \in \mathbb{R}^{2}, b_{i} \in \mathbb{R}^{2}, c_{j} \in \mathbb{R}^{2}\right\},
$$

where $I$ is the set of the indices of the classical finite element nodes and $I_{H}$ is the set of the indices of the nodes enriched by the following Heaviside function:

$$
H(x)= \begin{cases}1 & \text { if }\left(x-x^{*}\right) \cdot n \geqslant 0 \\ -1 & \text { elsewhere }\end{cases}
$$

$x^{*}$ denoting the crack tip and $n$ is a normal unit vector to the crack. Moreover, $\left(F_{j}, j=1, \ldots, 4\right)$ are singular functions given in polar coordinates by

$$
\left\{F_{j}(x)\right\} \equiv\left\{\sqrt{r} \sin \left(\frac{\theta}{2}\right), \sqrt{r} \cos \left(\frac{\theta}{2}\right), \sqrt{r} \sin \left(\frac{\theta}{2}\right) \sin (\theta), \sqrt{r} \cos \left(\frac{\theta}{2}\right) \sin (\theta)\right\},
$$

and $\gamma$ is a $C^{3}$ cut-off function such that $\left(0<r_{0}<r_{1}\right)$

$$
\gamma(x)=1 \quad \text { if } x<r_{0}, \quad 0<\gamma(x)<1 \quad \text { if } r_{0}<x<r_{1}, \quad \gamma(x)=0 \quad \text { if } r_{1}<x .
$$

The discrete problem can be written as follows

$$
\text { Find } u^{h} \in \vartheta^{h} \text { such that } a\left(u^{h}, v^{h}\right)=l\left(v^{h}\right) \quad \forall v^{h} \in \vartheta^{h} .
$$

\section{Error estimate}

Theorem 4.1. Let the displacement field $u$, solution to problem (1), satisfy the condition (2). Then, the following estimate holds

$$
\left\|u-u^{h}\right\|_{1, \Omega} \leqslant C h\left\|u-\gamma u_{s}\right\|_{2+\epsilon, \Omega},
$$

where $u^{h}$ is the solution to problem (8) and $C>0$ is a constant only depending on $\Omega$. 

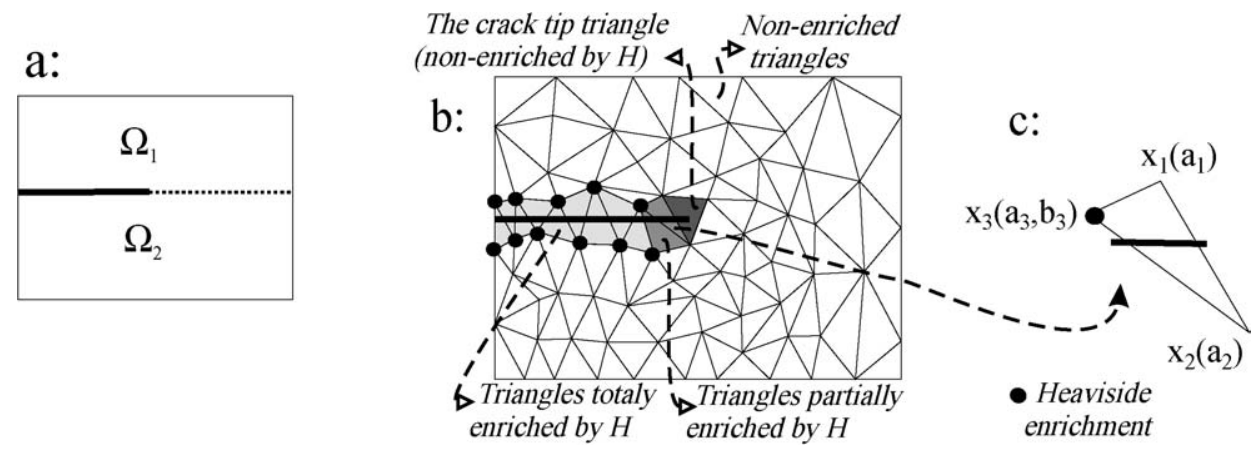

Fig. 1. The domain decomposition and the enrichment strategy.

Essential steps of the proof. The linear finite element method we consider is defined on a regular triangulation $\mathcal{T}_{h}$ of the non-cracked bi-dimensional domain $\bar{\Omega}$, where $h=\max _{K \in \mathcal{T}_{h}}\left(h_{K}\right)$ with $h_{K}=\operatorname{diam}(K)=\max _{x_{1}, x_{2} \in K}\left\|x_{1}-x_{2}\right\|$ $\left(K \in \mathcal{T}_{h}\right)$. Let $\rho_{K}=\left\{\sup (\operatorname{diam}(B)) ; B\right.$ ball of $\left.\mathbb{R}^{2}, B \subset K\right\}$. In order to define an interpolation operator, and since the displacement field is discontinuous across the crack, we divide $\Omega$ into two sub-domains $\Omega_{1}$ and $\Omega_{2}$ according to the crack and a straight extension of this crack (Fig. 1(a)). The displacement fields over $\Omega_{1}$ and $\Omega_{2}$ are denoted $u_{1}$ and $u_{2}$ respectively. Let $u_{r d}$ be the 'regular discontinuous' displacement defined by $u_{r d}=u-\gamma u_{s}$, thus $u_{1}$ and $u_{2}$ are the restrictions of $u_{r d}$ over $\Omega_{1}$ and $\Omega_{2}$ respectively. Let $\widetilde{u_{1}}$ and $\widetilde{u_{2}}$ be the extensions of $u_{1}$ over $\Omega_{2}$ and $u_{2}$ over $\Omega_{1}$ respectively such that (see [1]):

$$
\left\|\tilde{u_{1}}\right\|_{2+\epsilon, \bar{\Omega}} \leqslant C_{1}\left\|u_{1}\right\|_{2+\epsilon, \Omega_{1}} \quad \text { and } \quad\left\|\tilde{u_{2}}\right\|_{2+\epsilon, \bar{\Omega}} \leqslant C_{2}\left\|u_{2}\right\|_{2+\epsilon, \Omega_{2}} .
$$

Every triangle $K$ cut by the crack is divided into $K_{1}=K \cap \Omega_{1}$ (where $u_{1}$ lives) and $K_{2}=K \cap \Omega_{2}$ (where $u_{2}$ lives). In order to interpolate on $K_{1}$ (respectively on $K_{2}$ ) one needs three interpolation points, that is why the nodal values of the extension of $u_{1}$ (respectively $u_{2}$ ) over $K$ will be used. Thus every triangle totally enriched by $H$ will have twelve degrees of freedom. Note that a similar work has been done in [7], but for a domain totally cut by the crack, which means that the domain does not contain a crack tip.

We define now the interpolation operator for a function $u$ satisfying (2) as follows

$$
\Pi^{h} u=\sum_{i \in I} a_{i} \varphi_{i}+\sum_{i \in I_{H}} b_{i} H \varphi_{i}+\sum_{i=1}^{4} c_{i} F_{i} \gamma,
$$

such that $\left(x_{i}\right.$ denoting the node of index $\left.i\right)$ :

$$
\begin{aligned}
& \text { if } i \in I \backslash I_{H} \text { then } a_{i}=u\left(x_{i}\right), \\
& \text { if } i \in I_{H} \text { and if } x_{i} \in \Omega_{1} \text { then } a_{i}+b_{i}=u_{1}\left(x_{i}\right) \text { and } a_{i}-b_{i}=\tilde{u_{2}}\left(x_{i}\right), \\
& \text { if } i \in I_{H} \text { and if } x_{i} \in \Omega_{2} \text { then } a_{i}-b_{i}=u_{2}\left(x_{i}\right) \text { and } a_{i}+b_{i}=\tilde{u_{1}}\left(x_{i}\right) .
\end{aligned}
$$

Finally $c_{i}, i=1, \ldots, 4$, are defined such that $\sum_{i} c_{i} F_{i}=u_{s}$.

In order to find the global interpolation error, we will proceed by computing local error estimates over the triangles totally enriched by $H$, the triangle containing the crack tip, the triangles partially enriched by $H$ and the non-enriched triangles (Fig. 1(b)).

Lemma 4.2. Let $\mathcal{T}_{h}^{H}$ be the set of triangles totally enriched by $H$ (Fig. 1(b)) and $\sigma_{K}=\frac{h_{K}}{\rho_{K}}$. There exists a constant $C>0$ independent of $h$ such that, for all $K$ in $\mathcal{T}_{h}^{H}$ and for all $u$ satisfying (2), we have

$$
\left\|u-\Pi^{h} u\right\|_{1, K \cap \Omega_{1}} \leqslant C h_{K} \sigma_{K}\left\|\tilde{u_{1}}\right\|_{2+\epsilon, K} \text { and }\left\|u-\Pi^{h} u\right\|_{1, K \cap \Omega_{2}} \leqslant C h_{K} \sigma_{K}\left\|\tilde{u_{1}}\right\|_{2+\epsilon, K} .
$$

In fact, as we said before, the triangles totally enriched by $H$ are cut in two parts. Using the extensions of $u_{1}$ and $u_{2}$, we associate three interpolation points to every part. Thus the interpolation operator we defined allows us to make a classical interpolation over each part of the triangle, and to have the same optimal rate of convergence obtained in the classical global interpolation theorem (see $[2,4,12]$ ). Lemma 4.2 is a direct consequence of this theorem. 
Lemma 4.3. Let $K$ be the triangle containing the crack tip and $K^{*}=K \backslash \Gamma_{C}$. Using the same notations as before, we have the following estimate over $K^{*}$

$$
\left\|u-\Pi^{h} u\right\|_{1, K^{*}} \leqslant C h_{K} \sigma_{K}\left\|u-\gamma u_{s}\right\|_{2+\epsilon, K^{*}}
$$

Proof. Since we added singular functions to the discrete space (around the crack tip), the singular part of $u$ (see (2)) will be eliminated when we try to estimate $\left\|u-\Pi^{h} u\right\|_{1, K^{*}}$. All we have to do is to estimate $\left\|u_{r d}-\Pi^{h} u_{r d}\right\|_{1, K^{*}}$ where $u_{r d}=u-\gamma u_{s}$. In fact $\left\|u_{r d}-\Pi^{h} u_{r d}\right\|_{1, K^{*}}^{2}=\left\|u_{r d}-\Pi^{h} u_{r d}\right\|_{0, K^{*}}^{2}+\left|u_{r d}-\Pi^{h} u_{r d}\right|_{1, K^{*}}^{2}$, where $|\cdot|_{1}$ denotes the $H^{1}$ semi-norm. Using Sobolev imbedding theorems, the space $H^{2+\epsilon}\left(K^{*}\right)$ is continuously imbedded in $\mathcal{C}_{B}^{1}\left(K^{*}\right)$ (see [1]). Thus $\left\|\nabla u_{r d}\right\|_{\infty, K^{*}} \leqslant C\left\|u_{r d}\right\|_{2+\epsilon, K^{*}}=\alpha$ where $C$ is a generic constant, and $\left\|\nabla \Pi^{h} u_{r d}\right\|_{\infty, K^{*}} \leqslant 2 \alpha d \rho_{K}^{-1}$ where $d \leqslant 2 h_{K}$ denotes the maximal distance between a node of $K^{*}$ and the crack tip. In fact, $\left(u_{r d}-\Pi^{h} u_{r d}\right)\left(x_{i}\right)$ vanishes on the nodes, thus $\left\|u_{r d}-\Pi^{h} u_{r d}\right\|_{\infty, K^{*}} \leqslant 5 \alpha h_{K}^{2} \rho_{K}^{-1}$, then

$$
\left\|u_{r d}-\Pi^{h} u_{r d}\right\|_{0, K^{*}} \leqslant\left(\frac{C h_{K}^{3}}{\rho_{K}}\right)\left\|u_{r d}\right\|_{2+\epsilon, K^{*}}, \quad\left|u_{r d}-\Pi^{h} u_{r d}\right|_{1, K^{*}} \leqslant\left(\frac{C h_{K}^{2}}{\rho_{K}}\right)\left\|u_{r d}\right\|_{2+\epsilon, K^{*}} .
$$

Finally we obtain (14).

Lemma 4.4. Let $K$ be a triangle partially enriched by $H$ (Fig. 1(b)). Over this triangle, the interpolation error can be bounded as follows

$$
\left\|u-\Pi^{h} u\right\|_{1, K} \leqslant C \frac{h^{2}}{\rho_{K}}\left\|u-\gamma u_{s}\right\|_{2+\epsilon, K} .
$$

Proof. This lemma can be proven in a similar way to Lemma 4.3.

The convergence estimate over the non-enriched triangles can obviously be deduced from the classical interpolation theorem. Then, for every non-enriched triangle $K$ we have

$$
\left\|u-\Pi^{h} u\right\|_{1, K} \leqslant C h_{K} \sigma_{K}\left\|u-\gamma u_{s}\right\|_{2+\epsilon, K} .
$$

The result of Theorem 4.1 is obtained by calculating the sum of the local estimates (13), (14), (16) and (17), then by using Cea's lemma (see [2] for instance).

\section{Concluding remarks}

(i) It is not excluded that $u_{r d} \in H^{2+\epsilon}(\Omega)$ can be weaken to $u \in H^{2}(\Omega)$, but with more difficult computations of $\left\|u-u^{h}\right\|_{1, K}$ when $K$ is a triangle partially cut by the crack.

(ii) Let us note that the work presented in [12] is applied to a mesh respecting the crack geometry. Thus it does not involve the problem presented here of the triangles partially enriched by the Heaviside function. On the other hand, this note offers an improvement for the 'classical' XFEM method where the convergence rate remains of order $\sqrt{h}$ for some reasons detailed in [8].

\section{References}

[1] R.A. Adams, Sobolev Spaces, Academic Press, 1975.

[2] Ph.G. Ciarlet, The Finite Element Method For Elliptic Problems, North-Holland Publishing Company, 1979.

[3] P. Destuynder, M. Djaoua, S. Lescure, Une nouvelle méthode de calcul de la singularité pour un problème elliptique posé sur un ouvert avec fente, C. R. Acad. Sci. Paris 293 (1981) 343-346.

[4] A. Ern, J.L. Guermond, Eléments finis: théorie, applications, mise en œuvre, Springer, 2001.

[5] A. Gravouil, N. Moës, T. Belytschko, Non-planar 3D crack growth by the extended finite element and level sets, Part II: Level set update, Int. J. Numer. Methods Engrg. 53 (11) (2002) 2569-2586.

[6] P. Grisvard, Singularities in Boundary Value Problems, Masson, 1992.

[7] A. Hansbo, P. Hansbo, A finite element method for the simulation of strong and weak discontinuities in solid mechanics, Comput. Methods Appl. Mech. Engrg. 193 (2004) 3523-3540.

[8] P. Laborde, Y. Renard, J. Pommier, M. Salaün, High order extended finite element method for cracked domains, Int. J. Numer. Methods Engrg., in press. 
[9] J.B. Leblond, Mécanique de la Rupture Fragile et Ductile, Hermes, Lavoisier, 2003.

[10] J. Lemaitre, J.-L. Chaboche, Mechanics of Solid Materials, Cambridge University Press, 1994.

[11] N. Moës, J. Dolbow, T. Belytschko, A finite element method for crack growth without remeshing, Int. J. Numer. Methods Engrg. 46 (1999) $131-150$.

[12] G. Strang, G. Fix, An Analysis of the Finite Element Method, Prentice-Hall, Englewood Cliffs, 1973.

[13] F.L. Stazi, E. Budyn, J. Chessa, T. Belytschko, An extended finite element method with higher-order elements for curved cracks, Comput. Mech. 31 (2003) 38-48. 